\title{
11. Upward and Downward Continuation of Gravity Values Based on the Cylindrical Co-ordinate System
}

By Chuji TsuboI, M.J.A.

Geophysical Institute, Tokyo University

(Comm. Jan. 12, 1961)

Let in Fig. 1, $S$ be a horizontal underground plane which is situated at a depth $d$ from the earth's surface $O$ and a two-dimensional distribution of condensed mass $M$ is assumed on it. Gravity anomalies on the earth's surface which are due to this underground mass will be denoted by $\Delta g$.

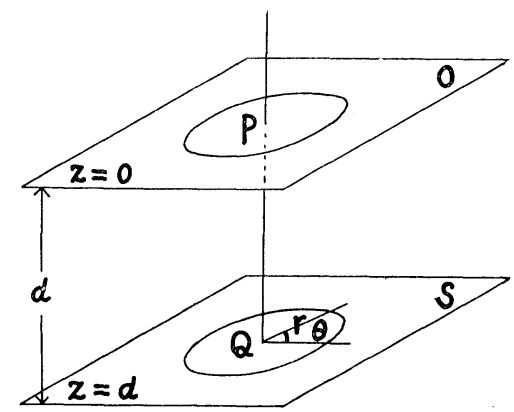

Fig. 1

Referring to the cylindrical co-ordinate system taken as shown in Fig. 1, $\Delta g$ and $M$ are expressed in Bessel-Fourier series as follows:

$$
\begin{aligned}
& \Delta g=\sum_{n} \sum_{\alpha} B_{n \alpha} J_{n}\left(\frac{\alpha r}{a}\right)_{\sin }^{\cos } n \theta, \\
& M=\frac{1}{2 \pi k^{2}} \sum_{n} \sum_{\alpha} B_{n \alpha} J_{n}\left(\frac{\alpha r}{a}\right){ }_{\sin }^{\cos n \theta \exp \frac{\alpha d}{a},}
\end{aligned}
$$

where $J_{n}$ is the Bessel function of $n$-th order, $a$ a unit for measuring distance, $\alpha$ a gauge parameter, and $k^{2}$ the universal constant of gravitation.

Mass at point $Q$, which is denoted by $M_{Q}$, is known by putting $r$ in (2) to be equal to zero. But since

$2 \pi k^{2} M_{Q}$ is simply expressed by

$$
\begin{aligned}
& J_{0}(0)=1, \\
& J_{1}(0)=J_{2}(0)=\cdots=0,
\end{aligned}
$$

$$
2 \pi k^{2} M_{Q}=\sum_{\alpha} B_{0 \alpha} \exp \frac{\alpha d}{a} .
$$

Referring to (1), $B_{0 \alpha}$ is seen to represent the coefficients in the following expression 


$$
\overline{\Delta g(r)}=B_{0 \alpha_{1}} J_{0}\left(\frac{\alpha_{1} r}{a}\right)+B_{0 \alpha_{2}} J_{0}\left(\frac{\alpha_{2} r}{a}\right)+B_{0 \alpha_{3}} J_{0}\left(\frac{\alpha_{3} r}{a}\right)+\cdots,
$$

which is a $J_{0}$ function series consisting of terms with different values of $\alpha$ and gives the average value of $\Delta g$ taken along a circle $r=r$ around point $P$ throughout the whole azimuth from 0 to $2 \pi$.

Equation (3) states that if the values of $B_{0 \alpha}$ in (4) could once be known, $M_{Q}$ will immediately be deduced as $1 / 2 \pi k^{2}$ of the sum of their products into corresponding $\exp \frac{\alpha d}{a}$. The number of terms which should be taken in the series (4) in order that it may be a good representation of the actual function $\overline{\Delta g(r)}$ depends on the accuracy of results which is required. In the present article, $\overline{\Delta g(r)}$ from $r=0$ up to $r=5 \alpha$ will be approximated by a series having six terms, although this tentative number as well as the limit of distance may be increased if desired.

In subsequent calculations, $\{\overline{\Delta g(r)}-\overline{\Delta g(5 a)}\}$ will be used instead of $\overline{\Delta g(r)}$ itself and correspondingly $\left\{2 \pi k^{2} M_{Q}-\overline{\Delta g(5 a)}\right\}$ instead of $2 \pi k^{2} M_{Q}$. If the difference $\{\overline{\Delta g(r)}-\Delta g(5 a)\}$ be expressed as follows:

$$
\begin{aligned}
& \overline{\Delta g(r)}-\overline{\Delta g(5 a)} \\
= & B_{0 \alpha} J_{0}\left(\frac{\alpha_{1} r}{a}\right)+B_{0 \alpha_{2}} J_{0}\left(\frac{\alpha_{2} r}{a}\right)+B_{0 \alpha_{3}} J_{0}\left(\frac{\alpha_{3} r}{a}\right) \\
+ & B_{0 \alpha} J_{0}\left(\frac{\alpha_{4} r}{a}\right)+B_{0 \alpha} J_{0}\left(\frac{\alpha_{5} r}{a}\right)+B_{0 \alpha_{6}} J_{0}\left(\frac{\alpha_{6} r}{a}\right),
\end{aligned}
$$

then (3) is reduecd to

$$
\left\{2 \pi k^{2} M_{Q}-\overline{\Delta g(5 a)}\right\}=\sum_{\alpha} B_{0 \alpha} \exp \frac{\alpha d}{a} .
$$

Since $\{\overline{\Delta g(r)}-\overline{\Delta g(5 a)}\}$ vanishes at $r=5 a, \alpha_{1}, \alpha_{2}, \cdots, \alpha_{6}$ in (5) must be such that $J_{0}\left(5 \alpha_{1}\right), J_{0}\left(5 \alpha_{2}\right), \cdots, J_{0}\left(5 \alpha_{6}\right)$ will also vanish. The values of $\alpha$ to be used are therefore $1 / 5$ of the first six roots of the equation $J_{0}(x)=0$, so that

$$
\begin{array}{ll}
\alpha_{1}=0.481, & \alpha_{4}=2.358, \\
\alpha_{2}=1.104, & \alpha_{5}=2.986, \\
\alpha_{3}=1.731, & \alpha_{6}=3.614 .
\end{array}
$$

Since the series (5) involves six coefficients $B_{0 \alpha}$ which are adjustable, it can be made to give values which coincide with observed values of $\{\overline{\Delta g(r)}-\overline{\Delta g(5 a)}\}$ at six different distances $r$ from the origin, including $r=0$.

A hexagonal mesh which is shown in Fig. 2 is supposed to be drawn on the earth's surface and the distance between the two neighboring grid points of it will be taken as the unit $a$ for measuring distance. Referring to this mesh, those points which are at distances $r=0, a, \sqrt{3 a}, 2 a, 3 a, 2 \sqrt{3 a}$, and $5 a$ from the origin will be considered. 


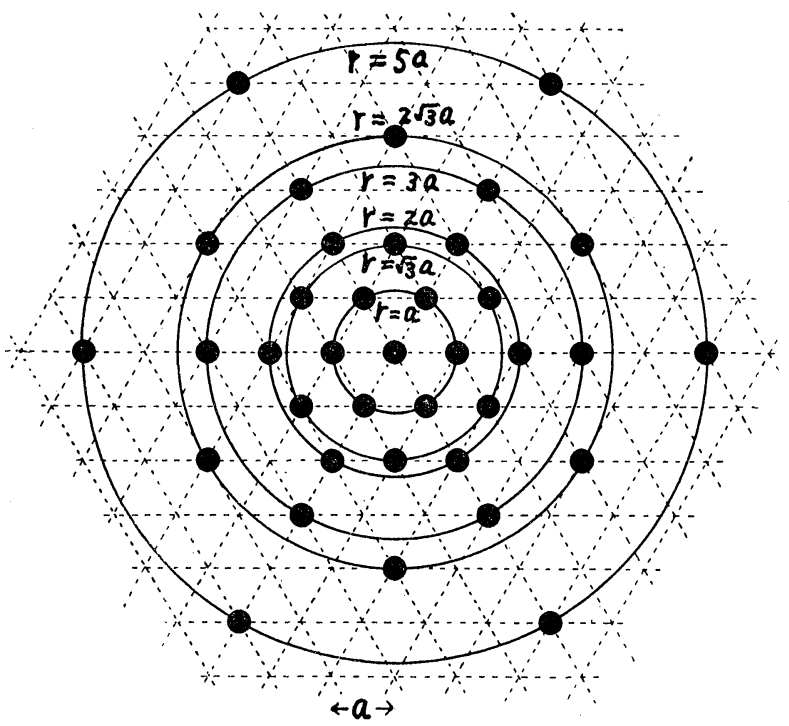

Fig. 2

$\overline{\Delta g(r)}$ value for each of these radii can be evaluated approximately by dividing by six the sum of $\Delta g$ values at six grid points which are shown in Fig. 2 by dots on the corresponding circle. In terms of these $\overline{\Delta g(r)}$ values, the following set of simultaneous equations is obtained for determining $B_{0 \alpha}$ values in (5):

$$
\left(\begin{array}{c}
\frac{\Delta g(0)}{\overline{\Delta g(a)}}-\overline{\Delta g(5 a)} \\
\overline{\Delta g(5 a)} \\
\overline{\Delta g(\sqrt{3} a)}-\overline{\Delta g(5 a)} \\
\overline{\Delta g(2 a)}-\overline{\Delta g(5 a)} \\
\overline{\Delta g(3 a)}-\overline{\Delta g(5 a)}
\end{array}\right)=A\left(\begin{array}{c}
B_{0 \alpha_{1}} \\
B_{0 \alpha_{2}} \\
B_{0 \alpha_{3}} \\
B_{0 \alpha_{4}} \\
B_{0 \alpha_{5}} \\
B_{0 \alpha_{6}}
\end{array}\right),
$$

where $A$ represents the following matrix:

$$
A=\left(\begin{array}{llllll}
1 & \multicolumn{1}{c}{1} & \multicolumn{1}{c}{1} & \multicolumn{1}{c}{1} & \multicolumn{1}{c}{1} & \multicolumn{1}{c}{1} \\
J_{0}\left(\alpha_{1}\right) & J_{0}\left(\alpha_{2}\right) & J_{0}\left(\alpha_{3}\right) & J_{0}\left(\alpha_{4}\right) & J_{0}\left(\alpha_{5}\right) & J_{0}\left(\alpha_{6}\right) \\
J_{0}\left(\sqrt{3} \alpha_{1}\right) & J_{0}\left(\sqrt{3} \alpha_{2}\right) & J_{0}\left(\sqrt{3} \alpha_{3}\right) & J_{0}\left(\sqrt{3} \alpha_{4}\right) & J_{0}\left(\sqrt{3} \alpha_{5}\right) & J_{0}\left(\sqrt{3} \alpha_{6}\right) \\
J_{0}\left(2 \alpha_{1}\right) & J_{0}\left(2 \alpha_{2}\right) & J_{0}\left(2 \alpha_{3}\right) & J_{0}\left(2 \alpha_{4}\right) & J_{0}\left(2 \alpha_{5}\right) & J_{0}\left(2 \alpha_{6}\right) \\
J_{0}\left(3 \alpha_{1}\right) & J_{0}\left(3 \alpha_{2}\right) & J_{0}\left(3 \alpha_{3}\right) & J_{0}\left(3 \alpha_{4}\right) & J_{0}\left(3 \alpha_{5}\right) & J_{0}\left(3 \alpha_{6}\right) \\
J_{0}\left(2 \sqrt{3} \alpha_{1}\right) & J_{0}\left(2 \sqrt{3} \alpha_{2}\right) & J_{0}\left(2 \sqrt{3} \alpha_{3}\right) & J_{0}\left(2 \sqrt{3} \alpha_{4}\right) & J_{0}\left(2 \sqrt{3} \alpha_{5}\right) & J_{0}\left(2 \sqrt{3} \alpha_{6}\right)
\end{array}\right) .
$$

With numerical values of $J_{0}$ functions being put into the equations, the solution of (6) is given as follows: 


$$
\left(\begin{array}{l}
B_{0 \alpha_{1}} \\
B_{0 \alpha_{2}} \\
B_{0 \alpha_{3}} \\
B_{0 \alpha_{4}} \\
B_{0 \alpha_{5}} \\
B_{0 \alpha_{6}}
\end{array}\right)=C\left(\begin{array}{r}
\overline{\Delta g(0)}-\overline{\Delta g(5 a)} \\
\overline{\Delta g(a)}-\overline{\Delta g(5 a)} \\
\overline{\Delta g(\sqrt{3} a)}-\overline{\Delta g(5 a)} \\
\overline{\Delta g(2 a)}-\overline{\Delta g(5 a)} \\
\overline{\Delta g(3 a)}-\overline{\Delta g(5 a)} \\
\overline{\Delta g(2 \sqrt{3} a)}-\overline{\Delta g(5 a)}
\end{array}\right),
$$

where $C$ is the reciprocal matrix of $A$ and is

$$
C=\left(\begin{array}{rrrrrr}
-0.130 & 0.816 & -1.737 & 2.131 & -0.377 & 0.832 \\
0.562 & -1.172 & 5.229 & -4.842 & 1.825 & -2.453 \\
-0.503 & 2.528 & -6.945 & 5.877 & -3.858 & 3.384 \\
0.972 & -2.541 & 7.419 & -7.648 & 4.489 & -2.930 \\
-0.540 & 2.309 & -8.829 & 8.568 & -3.019 & 1.602 \\
0.640 & -1.940 & 4.863 & -4.087 & 0.939 & -0.435
\end{array}\right) .
$$

If $d=a$, that is, if the spacing of grid points of the mesh is so taken as to be equal to the assumed depth $d$ of the mass, equation $\left(3^{\prime}\right)$ is reduced to

Combining $\left(3^{\prime \prime}\right)$ and $(7)$ and with

$$
2 \pi k^{2} M_{Q}-\overline{\Delta g(5 a)}=\sum_{\alpha} B_{0 \alpha} \exp \alpha .
$$

$$
\begin{array}{ll}
\exp \alpha_{1}=1.618, & \exp \alpha_{4}=10.448, \\
\exp \alpha_{2}=3.016, & \exp \alpha_{5}=19.807, \\
\exp \alpha_{3}=5.646, & \exp \alpha_{6}=37.115,
\end{array}
$$

the following equation is obtained:

$$
\begin{aligned}
& 2 \pi k^{2} M_{Q}-\overline{\Delta g(5 a)} \\
= & 21.724\{\Delta g(0)-\overline{\Delta g(5 a)}\} \quad-40.557\{\overline{\Delta g(a)}-\overline{\Delta g(5 a)}\} \\
+ & 55.753\{\overline{\Delta g(\sqrt{3} a)}-\overline{\Delta g(5 a)}\}-38.850\{\overline{\Delta g(2 a)}-\overline{\Delta g(5 a)}\} \\
+ & 4.785\{\overline{\Delta g(3 a)}-\overline{\Delta g(5 a)}\}-1.404\{\overline{\Delta g(2 \sqrt{3} a)}-\overline{\Delta g(5 a)}\},
\end{aligned}
$$

which is reduced to

$$
\begin{aligned}
M_{Q}=\frac{1}{2 \pi k^{2}} & \{21.724 \Delta g(0)-40.557 \overline{\Delta g(a)} \\
& +55.753 \overline{\Delta g(\sqrt{3} a)}-38.850 \overline{\Delta g(2 a)} \\
& +4.785 \overline{\Delta g(3 a)}-1.404 \overline{\Delta g(2 \sqrt{3} a)} \\
& -0.451 \overline{\Delta g(5 a)}\} .
\end{aligned}
$$

But since $\overline{\Delta g(r)}$ is given as $\frac{1}{6} \sum^{6} \Delta g(r),(9)$ is transformed to

$$
\begin{aligned}
M_{Q}=\frac{1}{2 \pi k^{2}} & \left\{21.724 \Delta g(0) \quad-6.759 \sum^{6} \Delta g(a)\right. \\
& +9.292 \sum^{6} \Delta g(\sqrt{3} a)-6.475 \sum^{6} \Delta g(2 a) \\
& +0.797 \sum^{6} \Delta g(3 a)-0.234 \sum^{6} \Delta g(2 \sqrt{3} a) \\
& \left.-0.075 \sum^{6} \Delta g(5 a)\right\},
\end{aligned}
$$


and this is the final expression which is practically useful for computing $M_{Q}$ from surface gravity anomalies. For points other than $Q$, shift the mesh appropriately and repeat the calculation as indicated by (10) each time. By this way, the underground mass distribution which is unknown may easily be deduced from corresponding gravity anomalies which are known.

This method of gravity continuation which is based on the cylindrical co-ordinate system has advantages over those that are based on the rectangular co-ordinate system, especially in virtue of the fact that the relevant domain taken into consideration in it is of a circular form. It has been thought a defect of the latter methods that the relevant domain taken in them, being a square, is not of a uniform extension from the point of interest in different azimuths, so that undesirable errors cannot be avoided to creep in the results. The new method based on the cylindrical co-ordinate system is entirely free from such an awkwardness. The accuracy of results obtainable by this method may be made higher, if desired, by increasing the number of circles along which $\Delta g$ values are read and also by increasing the number of $\alpha$ correspondingly. But in that case an increased tedious work of reading $\Delta g$ values at greater number of points will become needed and this is not desirable. There may be a compromise between these two controversies. Experience has shown that with six different radii up to $r=5 a$ and with six different values of $\alpha$, as in the case of the above instance, a reasonable accuracy can be obtained.

The above method for downward continuation can readily be modified to be applicable for upward continuation of gravity values to an elevation $a$ also. In this case, an expression corresponding to $\left(3^{\prime \prime}\right)$ is

$$
\Delta g_{a}=\sum_{\alpha} B_{0 \alpha} \exp (-\alpha),
$$

and by similar calculations as in the case of downward continuation, the final expression is obtained as follows:

$$
\begin{aligned}
\Delta g_{a} & =0.09884 \Delta g(0) \quad+0.06460 \sum^{6} \Delta g(a) \\
& -0.03052 \sum_{6}^{6} \Delta g(\sqrt{3} a)+0.05876 \sum^{6} \Delta g(2 a) \\
& -0.00226 \sum^{6} \Delta g(3 a)+0.01539 \sum^{6} \Delta g(2 \sqrt{3} a) \\
& +0.04428 \sum^{6} \Delta g(5 a) .
\end{aligned}
$$

Numerical tests which were made on several model distributions of gravity anomalies have proved that the proposed methods are feasible and yield results which are satisfactorily accurate for many practical problems of gravity continuation in both upward and downward directions. 